\title{
LA AUTOCOMPOSICIÓN COMO MECANISMO ADECUADO PARA RESOLVER/ADMINISTRAR LOS LITIGIOS DERIVADOS DE LA IMPLEMEN- TACIÓN DE LAS POLÍTICAS DE SALUD PÚBLICA
}

\author{
SELF-COMPOSITION AS A PROPER MECHANISM \\ FOR SOLVING / ADMINISTERING DISPUTES FROM \\ IMPLEMENTATION OF PUBLIC HEALTH POLICIES
}

\author{
Fabiana Marion Spengler ${ }^{1}$ Fernando A. Marion Spengler ${ }^{2}$ \\ Pedro Henrique Marion Spengler ${ }^{3}$
}

DOI: https://doi.org/10.37767/2591-3476(2020)02

\section{RESUMEN:}

El tema de este artículo es la autocomposición. El principal problema de investigación que debe responderse es el siguiente: ¿es la autocomposición un mecanismo adecuado para administrar/resolver los litigios derivados de la no implementación de las políticas de salud pública? La hipótesis responde afirmativamente al problema de la investigación. Del problema surge el objetivo: analizar la autocomposición como un procedimiento adecuado para administrar/resolver los litigios derivados de la no implementación de las políticas de salud pública. Se utilizó, el método deductivo como método de abordaje. Como método de procedimiento se utilizó el método monográfico y como técnica de investigación se aplicó la bibliográfica. La conclusión principal se refiere a la confirmación de la hipótesis en el sentido de que la autocomposición, ejemplificada por la mediación y la conciliación, es el mecanismo adecuado para administrar/resolver litigios derivados de la implementación y práctica de las políticas de salud pública.

\footnotetext{
1 Fabiana Marion Spengler es una investigadora fondeada por el CNPq (PQ2) en Productividad en Investigación, con posdoctorado en Derecho por la Università degli Studi di Roma Tre, en Roma, Italia, con beca CNPq (PDE). Doctorado en Derecho por el programa de Posgrado stricto sensu de la Universidade do Vale do Rio dos Sinos - UNISINOS - RS, con beca Capes, Maestría en Desarrollo Regional, con concentración en el área de Política Institucional de la Universidade de Santa Cruz do Sul - UNISC - RS, profesora de los cursos de Grado y Posgrado lato e stricto sensu de la UNISC, Coordinadora del Grupo de Investigación "Políticas Públicas en el Tratamiento de Conflictos" vinculado al CNPq; coordinadora del proyecto de investigación "El tercero y el conflicto: el mediador, el conciliador, el juez, el árbitro y sus roles políticos y sociales" financiado por la Fundación de Apoyo a la Investigación del Estado del Rio Grande do Sul - Fapergs, Edital 02/2017 - PqG - Investigadora Gaucha, coordinadora y mediadora del proyecto de extensión: "La crisis de jurisdicción y la cultura de la paz: la mediación como medio democrático, autónomo y consensual de resolución de conflictos" financiado por la Universidade de Santa Cruz do Sul - UNISC; autora de varios libros y artículos científicos; correo electrónico: fabiana@unisc.br; link Curriculum Lattes: http://lattes.cnpq.br/8254613355102364. ORCID iD: https://orcid.org/0000-0001-9477-5445.
}

2 Doctor graduado de la Universidade de Santa Cruz do Sul (UNISC). Miembro de la Liga de Neurología de la UNISC. Fue representante del Departamento Universitario de la Asociación Médica del Rio Grande do Sul (AMRIGS). Correo electrónico: fernandoamspengler@gmail.com. ORCID iD: https://orcid. org/0000-0003-3329-2866.

3 Estudiante del curso de Medicina de la Universidade de Santa Cruz do Sul (UNISC). Miembro de la Liga de Psiquiatria de la UNISC. Correo electrónico: pedro.spengler@hotmail.com. ORCID iD: https://orcid.org/0000-0001-6256-0378. 


\begin{abstract}
This article focuses on self-composition as a mechanism for managing / resolving disputes arising from public health policies. The main research problem to be answered is: is selfcomposition an adequate mechanism to manage / resolve disputes arising from the nonimplementation of public health policies? The main hypothesis answers the research problem in the affirmative. From the research problem is born the objective: to analyze self-composition as an appropriate procedure to manage / resolve disputes arising from the non-implementation of public health policies. To meet the proposed objective, the deductive method was used as approach method. As a method of procedure the monographic method was used and as a research technique the bibliographic method was applied. The main conclusion concerns the confirmation of the hypothesis that self-composition, exemplified by mediation and conciliation, is an appropriate mechanism to manage / resolve disputes arising from the implementation and practice of public health policies.
\end{abstract}

PALABRAS CLAVE: Autocomposición; Litigios; Mecanismos; Políticas Públicas; Salud.

KEY WORDS: Self-composition; Litigation; Mechanisms; Public policy; Health.

\title{
I. Introducción
}

La salud es un derecho humano garantizado a todos los brasileños, y el Estado es el responsable de su garantía a través de la implementación de políticas sociales y económicas, según lo determina el artículo 196 de la Constitución Federal de 1988. Sin embargo, el incumplimiento de este precepto constitucional genera conflictos que se envían al Poder Judicial en forma de litigios procesualizados.

Este hecho, sumado a la conocida explosión de litigiosidad que enfrenta Brasil, congestiona el Poder Judicial y cuestiona su eficiencia en la respuesta jurisdiccional. Es de conocimiento general, probado científicamente ${ }^{4}$, que el Poder Judicial brasileño es incapaz de responder, en términos cualitativos y cuantitativos, a las demandas judiciales que se le dirigen.

Así, en vista de la dificultad de ofrecer una asistencia jurisdiccional adecuada a los litigios legales en todas las esferas, incluidas las relacionadas con el Derecho a la Salud (litigios "salud legal"), se buscan caminos alternativos/complementarios para responder a las demandas, entre ellas la autocomposición.

Se observa que la autocomposición es un mecanismo razonablemente nuevo en el escenario jurídico brasileño, ya que está regulada por el Código de Procedimiento Civil y por la Ley 13140, ambos de 2015. En este sentido, este artículo tiene como tema la autocomposición como mecanismo para administrar o resolver los litigios derivados de las políticas de salud pública. El principal problema de investigación que debe responderse es el

4 El hecho de que el Poder Judicial no está satisfaciendo su demanda puede ser comprobado por la publicación anual del Informe Justicia en Números, de Consejo Nacional de Justicia - CNJ. El último informe señala una tasa de congestión (que es el "indicador que compara lo que no se redujo con lo que se procesó durante el año base - suma de los nuevos casos y de los casos pendientes iniciales)” de más del 70\%. Conselho Nacional de Justiça (2017): Relatório Justiça em Números. Tomado de: <http://www.cnj.jus.br/programas-e-acoes/pj-justica-em-numeros>, fecha de consulta: 13.08.2019. 
siguiente: ¿es la autocomposición un mecanismo adecuado para administrar/resolver los litigios derivados de la no implementación de las políticas de salud pública? La hipótesis principal responde afirmativamente al problema de investigación.

El problema de investigación da lugar al objetivo: analizar la autocomposición como un procedimiento adecuado para administrar/resolver los litigios derivados de la no implementación de las políticas de salud pública.

Para cumplir con el objetivo propuesto se utilizó, como método de abordaje, el deductivo, partiendo de la relación entre los argumentos generales, llamados premisas, para los argumentos particulares, hasta llegar a una conclusión. Como método de procedimiento se utilizó el método monográfico y como técnica de investigación se aplicó la bibliográfica, a partir de la lectura y registro de fuentes bibliográficas vinculadas al tema de la investigación.

El texto se divide en dos puntos, el primero de los cuales se refiere a las políticas públicas. En este punto se reservó un primer momento para analizar el concepto y la formulación de las políticas públicas en Brasil para luego abordar la judicialización ${ }^{5}$ de las llamadas políticas de salud.

Posteriormente, el texto discute la posibilidad de utilizar la autocomposición para tratar los litigios que surjan de la implementación o aplicación de las políticas de salud pública. En este punto, la mediación y la conciliación se analizan como formas autocompositivas de administración/resolución de tales litigios.

\section{1- Políticas de salud pública en Brasil}

Como se dijo anteriormente, la salud es un derecho de todos los brasileños y está contenida en la Constitución Federal actual. Es un derecho humano fundamental que tiene por objetivo promover y proteger la calidad de vida humana ${ }^{6}$.

En estos términos, la salud es considerada un bien humano resultante de la organización social, que consta de múltiples factores; por lo tanto, ganó espacio legislativo constitucional e infraconstitucional, estando en el centro del debate mundial cuyo objetivo es su garantía y protección. Para cumplir estos objetivos, se requiere que el "Estado asuma la responsabilidad de una política de salud integrada con otras políticas sociales y económicas y asegure su implementación7. Esto sucede a través de la implementación de políticas de salud pública que garanticen programas efectivos para su promoción.

En general, la expresión "política pública" se define como un programa o marco de acción gubernamental, porque consiste en un conjunto de medidas articuladas (coordinadas), cuyo propósito es dar impulso, es decir, mover la máquina del gobierno, hacia lograr algún objetivo de orden público o, desde el punto de vista del jurista, lograr un derecho ${ }^{8}$.

\footnotetext{
5 Es importante señalar que se utilizarán datos numéricos para ilustrar la judicialización de la salud. Estos datos se refieren al Estado de São Paulo. La elección por el estado de São Paulo es debido a la organización y accesibilidad de los datos.

6 MARTINI, Sandra Regina; STURZA, Janaína Machado (2019): Direitos Humanos: saúde e fraternidade, Evangraf, Porto Alegre.

7 CAMPOS, Gastão Wagner; BARROS, Regina Benevides de; CASTRO, Adriana Miranda de (2019): Avaliação de política nacional de promoção da saúde: ciencia e saúde coletiva, p. 746 Tomado de: http://www.cienciaesaudecoletiva.com.br, fecha de consulta: 19.08.2019.

8 BUCCI, Maria Paula Dallari (2006): “O conceito de política pública em direito” en BUCCI, Maria Paula Dallari (org.): Políticas públicas: reflexões sobre o conceito jurídico, Saraiva, São Paulo, p. 14.
} 
Una definición "funcional" de política pública es: "la policy-making se refiere fundamentalmente a los actores rodeados de restricciones que tratan de conciliar los objetivos políticos (policy goals) con los medios políticos (policy means), en un proceso que puede caracterizarse como 'resolución aplicada de problemas'". Los autores van más allá y definen que la identificación de los problemas y la aplicación de las soluciones encontradas (por imperfectas que sean) implican la articulación de objetivos políticos a través de deliberaciones y discursos, además del uso de herramientas políticas (policy tools) en un intento de alcanzar estos objetivos ${ }^{9}$.

Con el fin de estudiar las políticas públicas como un proceso aplicado a la resolución de un determinado problema, hay tres puntos centrales que pueden resumirse de la siguiente manera: "a) el agente primario de la policy-making pública es un agente del gobierno; b) la policy-making implica una decisión fundamental por parte de los gobiernos de hacer algo o no con respecto a un problema, y esta decisión es tomada por los políticos electos y otros funcionarios oficiales". Por lo tanto, es una decisión tomada para emprender un curso de acción particular; “c) la política pública es una determinación consciente de un gobierno"10.

Se puede decir que el uso del término política pública puede designar no solo "la política del Estado, sino la política del público, de todos". Se trata "de la política destinada a avanzar en los objetivos colectivos de mejora de la comunidad y cohesión - o de la interdependencia - social"11. En resumen, también se afirma que la política pública: "es un conjunto de decisiones y acciones adoptadas por organismos públicos y organizaciones de la sociedad, intencionalmente coherentes entre sí, que, bajo la coordinación estatal, están destinadas a enfrentar un problema político"12.

Toda política pública es una forma de intervención en las relaciones sociales para hacer frente a un problema político. Esta acción está siempre condicionada por los intereses y expectativas de los miembros de las relaciones. En consecuencia, "puede definirse como la búsqueda explícita y racional de un objetivo, gracias a la adecuada asignación de medios que, a través de un uso razonable, deben producir consecuencias positivas"13.

En el caso de la política pública de promoción de la salud, por ejemplo, se observa su papel en el cumplimiento, entre otros, de un objetivo específico: "crear servicios y acciones de salud que pongan a los sujetos - usuarios y profesionales de la salud -, como protagonistas en la organización del proceso productivo de salud, entendiendo que allí se produce salud, sujetos, mundo ${ }^{14 "}$.

Para implementarse, la política pública de promoción de la salud requiere la asignación de medios, como recursos humanos, capacitación adecuada y estructura, por parte de la administración pública. Por lo tanto, se puede señalar como una política pública, ya que

\footnotetext{
9 HOWLETT, Michael; RAMESH, M.; PERL, Anthony (2013): Política Pública, seus ciclos e subsistemas, uma abordagem integral, Elsevier, Rio de Janeiro, p. 5-6. 10 HOWLETT, Michael; RAMESH, M.; PERL, Anthony (2013): Op. Cit., p. 6-7.

11 MASSA ARZABE, Patrícia Helena (2006): “Dimensão jurídica das políticas públicas” en BUCCI, Maria Paula Dallari (org.): Políticas públicas: reflexões sobre o conceito jurídico, Saraiva, São Paulo, p. 60.

12 SCHMIDT, João Pedro (2018): “Para estudar políticas públicas: aspectos conceituais, metodológicos e abordagens teóricas”, en Revista do Direito, v. 3, n. 56, p. 119-149, sep/dic. 2018, Santa Cruz do Sul, p. 127.

13 GRAU, Eros Roberto (2000): O direito e o direito pressuposto, Malheiros, São Paulo, p. 26.

14 Es importante destacar que el autor utiliza el SUS (Servicio Único de Salud) como uno de los ejemplos de la política pública de promoción de la salud en Brasil. CAMPOS, Gastão Wagner; BARROS, Regina Benevides de; CASTRO, Adriana Miranda de (2019): Op. Cit., p.746.
} 
se refiere a un "conjunto de programas de acción gubernamental estable en el tiempo, racionalmente configurados, implementados y evaluados, dirigidos a la realización de derechos y objetivos social y jurídicamente relevantes". Estos conjuntos de programas de gobierno se identifican con la "distribución y redistribución de bienes y posiciones que brinden oportunidades para que cada persona pueda vivir con dignidad y ejercer sus derechos", a fin de asegurarles recursos y condiciones para la acción, así como la libertad de elección para el uso de estos recursos ${ }^{15}$.

Además, la política pública de promoción de la salud se clasifica como política de Estado, ya que expresa "opciones ampliamente apoyadas por las fuerzas políticas y sociales, tiene disposiciones legales y cuenta con mecanismos y regulaciones para su implementación". Las políticas de Estado "cruzan" gobiernos de diferentes concepciones ideológicas, precisamente porque están arraigadas en el aparato estatal y tienen una amplia legitimación. Caracterizar una política como "de Estado" no es conferir un "calificativo de excelencia ético-política, sino reconocer que cumple las condiciones para extenderse en el tiempo. Por lo tanto, a diferencia de las políticas de gobierno, que 'tienden a ser provisionales', las políticas de Estado son "duraderas"'16.

Para lograr sus objetivos, las políticas públicas se formulan en un proceso que abarca todo el procedimiento de discusión, aprobación e implementación. Particularmente en los sistemas democráticos, estos procesos se desarrollan en un escenario político en el que actúan diversos y distintos "actores políticos" (o "jugadores", en referencia específica a la teoría de los juegos). Los jugadores incluyen actores estatales oficiales y políticos profesionales (presidentes, líderes de partidos, legisladores, jueces, gobernadores, burócratas), así como grupos privados, sindicatos, medios de comunicación y otros miembros de la sociedad civil. Dichos actores interactúan en diversos "ámbitos", que pueden ser formales (como el Legislativo), o informales ("la calle"), y pueden tener distintos grados de transparencia ${ }^{17}$.

La teoría de los ciclos enumera "cinco fases que permiten comprender como surge y se desarrolla una política: (i) percepción y definición del problema; (ii) inserción en la agenda política; (iii) formulación; (iv) implementación; y (v) evaluación"18. La etapa de formulación de políticas públicas se basa en "estudios previos y en un sistema de información adecuado, definiendo no sólo los objetivos, sino también los recursos y el horizonte temporal de la actividad de planificación"19. En el caso de las políticas públicas dirigidas a la sociedad, el proceso de formulación de estas debe estar atento al contexto en el que se encuentra, buscando información para su adecuada implementación.

Además, la formulación de políticas públicas como actividad de planificación debe tener siempre en cuenta el objetivo que se pretende alcanzar y el propósito deseado. En el caso de la política pública de promoción de la salud, puede calificar, por ejemplo, las acciones del Sistema Único de Salud (SUS), reorganizando los servicios de salud, preparando "Ios profesionales de la salud que trabajan allí para incluir la población en la cons-

\footnotetext{
15 MASSA-ARZABE, Patrícia Helena (2006): Op. Cit., p. 63.

16 SCHMIDT, João Pedro (2018): Op. Cit., p. 127.

17 STEIN, Ernesto (2007): A política das políticas públicas: progresso econômicos e social na América Latina: relatório 2006, Banco Interamericano de Desenvolvimento e David Rockefeller Center for Latin America Studies, Havard University, Elsevier Washington, Rio de Janeiro, p. 17. 
trucción y gestión de sus proyectos de salud y procesos de trabajo, montando colegiados administradores que no se dejen burocratizar" etc. ${ }^{20}$.

Sin embargo, cuando las políticas de salud pública no se cumplen pueden generar conflictos que, mal organizados y administrados, terminan por llegar al Poder Judicial en la forma de litigios procesualizados (procesos) ${ }^{21}$. Estos procesos terminan contribuyendo a la congestión del Poder Judicial, además de recibir prestación jurisdiccional a menudo tardía e inadecuada para el tipo de demanda propuesta en ellos ${ }^{22}$.

En el estudio "Judicialización de la Política de Salud Pública en los Municipios Brasileños: Un Retrato Nacional" organizado por el CNJ (2015) se destaca "la necesidad de que el Poder Judicial establezca medios de formación que permitan a los actores políticos, legales y participativos involucrados en la resolución del conflicto transitar en el área de salud". Las capacidades señaladas son "competencias y habilidades prácticas de mediación, construcción de consensos y diálogo institucional centrados en la salud". Así, llama la atención el hecho de que el mencionado Informe señala que el área de salud debe "reconocer la necesidad de extrapolar el ámbito de la acción judicial, lo que requiere alternativas institucionalizadas que también incluyan el ámbito político y el de otras entidades involucradas"23.

La propuesta de que los litigios legales de salud encuentren otras vías para su administración/resolución va en la misma dirección del propuesto en otras áreas del Derecho. Desde 2010 un número creciente de litigios se han sometidos a medios autocompositivos, incluida la mediación. La mediación es un mecanismo de autocomposición también propuesto en el Informe de Judicialización de la Política de Salud Pública en los Municipios Brasileños: Un Retrato Nacional, del CNJ, con fecha de 2015, como un medio para responder adecuadamente a los litigios legales de salud.

\section{2- "Autocomposición legal de la salud 24"}

La autocomposición, un acto volitivo de las partes en la dirección de resolver el conflicto o litigio, puede ocurrir a través de la mediación, de la conciliación y de la negociación, con o sin la participación de un tercero imparcial, que podrá ayudar en este proceso. Esta es una forma autónoma (las partes son los titulares del poder para decidir la disputa) de administrar/resolver litigios, actuando de manera más eficiente para comprometer los intereses. Se basa en factores persuasivos y consensuales, por los cuales las partes

20 CAMPOS, Gastão Wagner; BARROS, Regina Benevides de; CASTRO, Adriana Miranda de (2004): Op. Cit., p. 746.

21 El municipio de São Paulo, por ejemplo, "actualmente cuenta con 51 mil demandas judiciales en curso y más de 35 mil demandas administrativas. Para manejar una cantidad tan grande de información, se está utilizando un sistema de información específico para la obtención de datos, el S-Codes. A partir del sistema, los gestores pudieron crear un índice de judicialización de São Paulo, que consiste en el número de demandas judiciales por cada 10 mil habitantes. El promedio de procesos en el Estado es de 3.3, pero en grandes regiones que se consideran centros de producción de conocimiento de salud, como Ribeirão Preto, Barretos y Marília, por ejemplo, hay un promedio de 11 procesos por cada diez mil habitantes. En el gran São Paulo el promedio es de 0.77”. Estos procedimientos incluyen: " $58 \%$ de las demandas judiciales provienen de la prescripción de un médico privado; $65 \%$ se refiere a medicamentos; $78 \%$ de ellos están estandarizados por el SUS; $2 \%$ son productos importados sin registro en la Agencia Nacional de Vigilancia Sanitaria ANVISA; el 22\% de las demandas requieren una marca comercial específica del medicamento." SETOR SAƯDE (2015): Judicialização da saúde no Brasil: dados e experiência. Tomado de: https://setorsaude.com.br/estudo-revela-situacao-da-judicializacao-da-saude-no-brasil/, fecha de consulta: 19.08.19.

22 En más del $80 \%$ de los procesos ( 8 de cada 10), la solicitud se concede automáticamente y rara vez trae la comprobación de la demanda y el uso por parte del paciente, o incluso el comprobante de entrega del medicamento. Sector salud (2015): Op. Cit.

23 RIBEIRO, Wesllay Carlos (2018): "A mediação como meio de resolução de conflitos na área de saúde, en Revista De Direito Sanitário: Jornaul of Health Law, USP, São Paulo, v.18, n.3, nov.2017/feb.2018, p. 65. Tomado de: https://www.revistas.usp.br/rdisan/article/view/144648\&gt, fecha de consulta: 16.09.2019.

24 La expresión aquí referida fue utilizada por el profesor Celso Fernando Campilongo en una conferencia dada en el Centro de Estudios e Investigaciones en Derecho de Salud -CEPEDISA - de la Facultad de Salud Pública de la Universidade de São Paulo - USP, el 28 de junio de 2019. 
componen el litigio, de tal manera que obtienen soluciones más duraderas y factibles ${ }^{25}$.

Se denomina autocomposición en la medida en que los involucrados en el litigio asumen el riesgo y la responsabilidad de la decisión que adoptan, por consenso, poniendo fin mediante una transacción (acuerdo), revocación (renuncia al derecho), sumisión (reconocimiento jurídico de la solicitud), etc.

El principal efecto de la autocomposición "es hacer desaparecer el litigio. Si es judicial, provoca el fin del proceso; si es preventivo, lo evita. Los alcances de la autocomposición son los mismos que los del proceso, de carácter jurídico, social y político, tanto en relación con los involucrados como, indirectamente, con la sociedad"26.

La principal diferencia entre autocomposición y heterocomposición se refiere al hecho de que, mientras que en los procesos heterocompositivos, cuyos modelos se denominan adversariales (arbitraje y jurisdicción), siempre hay ganadores y perdedores (ganadores/ perdedores), en los procesos autocompositivos de modelos consensuales (negociación, mediación y conciliación), se buscan las soluciones ganadoras (ganadores/ganadores), observando los intereses de todos ${ }^{27}$.

Precisamente por eso, la autocomposición puede ser un camino adecuado de tratar los litigios de la salud, ya que trabaja con el diálogo y con la búsqueda de consenso por parte de los propios litigantes. Aunque existen proyectos e iniciativas prácticas para la mediación en salud ${ }^{28}$ ésta es una de las formas autocompositivas, junto con la conciliación. Esta información es importante ya que, dependiendo del tipo de litigio y de sus características principales, se aplica la mediación o la conciliación.

Las diferencias entre la mediación y la conciliación son significativas. Ocurren en términos del concepto mismo, pero también operan significativamente en el papel desempeñado por los profesionales que administran las sesiones, el tipo de litigio tratado en ellas, los objetivos perseguidos y los resultados deseados ${ }^{29}$.

La mediación es un procedimiento de transformación de los antagonismos en puntos de convergencia (no necesariamente en puntos de acuerdo), a través de la intervención de un tercero elegido por las partes en conflicto. La mediación consiste en uno "de los instrumentos de pacificación de naturaleza autocompositiva y voluntaria, en el que un tercero, imparcial, actúa, de manera activa o pasiva, como facilitador del proceso de rea-

\footnotetext{
25 MORAIS, José Luis Bolzan de; SPENGLER, Fabiana Marion (2019): Mediação e arbitragem: Alternativas à jurisdição, Livraria do Advogado, Porto Alegre. 26 CAHALI, Francisco José (2015): Curso de arbitragem: mediação, conciliação, resolução, CNJ 125/2010, Revista dos Tribunais, São Paulo, p. 43.

27 BACELLAR, Roberto Portugal (2011): “O Poder Judiciário e o paradigma da guerra na solução de conflitos" en PELUSO, Antonio Cezar; RICHA, Morgana de Almeida (coord.): Conciliação e mediação: estruturação da Política Judiciária Nacional, Forense, Rio de Janeiro.

$28 \mathrm{Al}$ respecto, se puede citar la práctica de la mediación de la salud llevada a cabo "instituida en 2012 en el Ministerio Público del Estado de Minas Gerais, que consiste en una serie de acciones dirigidas a la confrontación de problemas colectivos en el área de la salud. Similar a la audiencia pública - evento que culmina en la realización de una reunión organizada, abierta a todos los ciudadanos, con el objetivo de "discutir, escuchar y recoger sugerencias, demandas e informaciones de la sociedad y de las autoridades públicas", proporcionando subsidios para una decisión administrativa (Resolución PGJ n. 29, del 25.03.2014, publicada en el Diario Oficial Electrónico del MPMG del 26.03.2014) - la Mediación Sanitaria fue concebida con un alcance resolutivo, proponiendo afrontar "complejas demandas en salud, con repercusión colectiva, especialmente en el aspecto preventivo, para encontrar soluciones consensuadas, substituyendo la judicialización evitable de la política de salud, con mayor legitimidad y efectividad" (Resolución PGJ n. 78, del 18.09.2012, publicada en el Diario Oficial Electrónico del MPMG del 20.09.2012) FERNANDES, Antonio Joaquim Schellenberger (2018): "Mediação Sanitária: Os elementos materiais do direito coletivo à saúde", en FERNANDES, Antonio Joaquim Schellenberger: Direito à saúde: tutela coletiva e mediação sanitária, Editora D’Plácido, Belo Horizonte, p. 368.

29 SPENGLER, Fabiana Marion (2019a): Dicionário de Mediação: Volume I, Essere nel Mondo, Santa Cruz do Sul.
} 
nudación del diálogo entre las partes, antes o después de que se establezca el conflicto ${ }^{30 "}$ . El mediador tiene, entonces, la tarea de permitir que las partes en conflicto expresen sus sentimientos y sus intereses dándoles espacio para formular de manera creativa sugerencias y propuestas para la resolución apropiada de su conflicto. El mediador no desempeña un papel activo en la propuesta de acuerdos, ni en la orientación de las partes en el conflicto. Esta tarea no es su responsabilidad ${ }^{31}$.

La conciliación, por su parte, es un instituto que tiene como objetivo alcanzar voluntariamente un acuerdo neutral y cuenta con la participación de un tercero - conciliador - que interviene, e incluso puede sugerir propuestas con el fin de dirigir la discusión. En la conciliación el objetivo es el acuerdo. En ella, las partes, incluso las adversarias, deben llegar a un acuerdo para evitar el proceso judicial o ponerle fin, si ya existe ${ }^{32}$. Precisamente por este motivo, el conciliador sugiere, orienta, interfiere y asesora a las partes, sin analizar el litigio en profundidad.

Al delimitar conciliación y mediación, se observa que la diferencia básica entre ambas está en el origen del litigio (relación continuada o relación esporádica), en la postura del mediador/conciliador o en las técnicas utilizadas por él33. Además, el objetivo principal de la conciliación es la pacificación de los litigios procesales mediante la elaboración de acuerdos. La mediación, por su parte, tiene como objetivo humanizar el conflicto, restaurando la comunicación rota entre los litigantes y hacerla viable y, si es posible, armoniosa. El acuerdo es un efecto secundario de la mediación y no el objetivo principal que debe perseguirse, como es el caso de la conciliación.

En resumen y de manera muy objetiva, la mediación y la conciliación difieren ${ }^{34}$ :

a) en cuanto al litigio: en la conciliación existen litigios esporádicos, sin que exista una relación previa o posterior entre las partes en conflicto. Ej.: relaciones de consumo entre los planes de salud y sus asegurados; mientras que en la mediación hay litigios en los cuales los involucrados tienen relaciones cercanas antes del problema y que permanecerán (al menos eso se espera), íntegras después de su resolución. Ejemplo típico son los litigios entre el médico y el paciente o entre el médico y el hospital, entre otros.

b) en cuanto al papel del mediador/conciliador: en la mediación hay una tercera persona que actúa como mediador, ayudando a las partes en conflicto a restablecer la comunicación. El mediador no sugiere, no propone, no guía. El conciliador, por el contrario, es el tercero que puede sugerir, proponer, guiar y dirigir el debate y sus resultados.

c) en cuanto a los objetivos perseguidos: la mediación busca una administración/resolución adecuada del litigio que pueda generar comunicación y satisfacción de los involucrados. El acuerdo puede ser una consecuencia de esta dinámica, pero un procedimiento de mediación que no haya concluido con un acuerdo no podrá calificarse de infructuoso si permitió que las partes en conflicto vuelvan a hablar. La mediación busca el consenso y la pacificación social efectiva. La conciliación tiene como objetivo principal llegar a un acuerdo, por lo que es un procedimiento centrado en la necesaria composi-

\footnotetext{
30 CAHALI, Francisco José (2015): Op. Cit., p. 85..

31 SPENGLER, Fabiana Marion (2019b): Dicionário de Mediação: Volume II, Essere nel Mondo, Santa Cruz do Sul.

32 CAHALI, Francisco José (2015): Op. Cit., p. 86.

33 CAHALI, Francisco José (2015): Op. Cit., p. 87.

34 SPENGLER, Fabiana Marion (2017): Mediação de conflitos. Teoria e Prática, Livraria do Advogado, Porto Alegre.
} 
ción entre las partes. El objetivo es resolver el litigio mediante un acuerdo.

d) en cuanto a las técnicas utilizadas y la dinámica de las sesiones: la mediación prevé el empleo de técnicas destinadas a escuchar y revelar el interés real involucrado en el litigio. Es un procedimiento dirigido a las personas que se convierten en protagonistas de sus historias y de la construcción de respuestas a sus problemas. La mediación prevé sesiones más largas que la conciliación, así como la reprogramación de dichas sesiones cuando sea necesario que el diálogo se mantenga y madure, de modo que posteriormente se puedan tomar las decisiones. Por otra parte, la conciliación prevé el uso de técnicas de negociación más centradas en el propio acuerdo; estimula las propuestas y contrapropuestas de las partes en conflicto y del conciliador. La conciliación se realiza en sesiones más cortas y la hipótesis de reprogramación de nuevas reuniones no es tan frecuente.

En vista de lo anterior, se observa que la mediación y la conciliación son institutos relacionados, aunque diferentes. Cada uno de ellos tiene sus límites y posibilidades al servicio de litigios específicos, con características y formas de acción también diferenciadas.

Así, cuando se propone un camino diferente para tratar los litigios jurídicos en materia de salud, cuyo objetivo es una respuesta rápida y de calidad, evadiendo la judicialización, es necesario verificar qué procedimiento autocompositivo es más apropiado: mediación o conciliación.

Ese cuidado es especialmente necesario cuando se trata de un litigio jurídico en materia de salud que tiene, en uno de los polos, la administración pública, por ejemplo. En este caso, según la Ley 13140/2015, en su art. 135, la autocomposición puede usarse para administrar/resolver el problema. La misma Ley determina la confidencialidad en el procedimiento de mediación y, dado que la administración pública está de uno de los lados de la disputa, es necesario buscar otro camino autocompositivo: la conciliación. Esto se debe a que uno de los principios de la administración pública es precisamente la publicidad, lo que es incompatible con uno de los principios básicos de la mediación que es precisamente la confidencialidad.

\section{Conclusión}

Con el fin de cumplir el precepto constitucional del derecho fundamental a la salud en Brasil, se crearon políticas públicas. Dichas políticas públicas, cuando no se cumplen pueden conducir a litigios. La mayoría de las veces estos litigios son direccionados al Poder Judicial, el cual, al resolverlos, no siempre ofrece una respuesta rápida, factible y/o adecuada.

Por lo tanto, en vista de la complejidad de los litigios relacionados con las cuestiones jurídicas de salud y de las dificultades cada vez más frecuentes de la jurisdicción para responder a esos litigios, se buscan caminos alternativos y/o complementarios. Uno de esos caminos es la autocomposición.

La autocomposición no es un procedimiento nuevo, sin embargo, ganó notoriedad en Brasil principalmente después de la promulgación del Código de Procedimiento Civil y de

35 Artículo 1: Esta Ley establece la mediación como medio de resolución de litigios entre particulares y sobre la autocomposición de conflic ᄀtos en el ámbito de la administración pública. 
la Ley 13140, ambos de 2015. Los mecanismos autocompositivos tienen como objetivo el consenso nacido del diálogo entre los litigantes ya que no funciona con la decisión del juez como un tercero que decide el litigio. La mediación y la conciliación son dos ejemplos de mecanismos autocompositivos.

De esta manera, la autocomposición es posible y totalmente factible para administrar/ resolver litigios jurídicos en materia de salud. Sin embargo, es necesario analizar el tipo de litigio y luego concluir sobre el mejor medio autocompositivo: ya sea mediación o conciliación.

La mediación servirá para conflictos que tengan un sesgo más afectivo y sentimental, en el que existan relaciones previas y continuas entre los involucrados, como las que existen entre el médico y su paciente. La conciliación ya se enfoca en el acuerdo, no teniendo como objetivo principal el mantenimiento o el restablecimiento de la relación rota. En estos casos, ella tiene un uso especial en los asuntos que impliquen, por ejemplo, las relaciones de consumo derivadas de los planes de salud.

En conclusión, puede observarse que la autocomposición puede ser utilizada para tratar litigios legales de salud considerando las cuestiones específicas que involucren el caso concreto y especialmente, la protección y promoción del derecho a la salud de todos los brasileños.

\section{REFERENCIAS BIBLIOGRÁFICAS}

- BACELLAR, Roberto Portugal (2011): “O Poder Judiciário e o paradigma da guerra na solução de conflitos" en PELUSO, Antonio Cezar; RICHA, Morgana de Almeida (coord.): Conciliação e mediação: estruturação da Política Judiciária Nacional, Forense, Rio de Janeiro. - BUCCI, Maria Paula Dallari (2006): "O conceito de política pública em direito" en BUCCI, Maria Paula Dallari (org.): Políticas públicas: reflexões sobre o conceito jurídico, Saraiva, São Paulo.

- CAHALI, Francisco José (2015): Curso de arbitragem: mediação, conciliação, resolução, CNJ 125/2010, Revista dos Tribunais, São Paulo.

- CAMPOS, Gastão Wagner; BARROS, Regina Benevides de; CASTRO, Adriana Miranda de (2019): Avaliação de política nacional de promoção da saúde: ciencia e saúde coletiva. Tomado de: http://www.cienciaesaudecoletiva.com.br, fecha de consulta: 19.08.2019.

- Conselho Nacional de Justiça (2017): Relatório Justiça em Números. Tomado de: <http://www.cnj.jus.br/programas-e-acoes/pj-justica-em-numeros>, fecha de consulta: 13.08.2019.

- GRAU, Eros Roberto (2000): O direito e o direito pressuposto, Malheiros, São Paulo.

- HOWLETT, Michael; RAMESH, M.; PERL Anthony (2013): Política Pública, seus ciclos e subsistemas, uma abordagem integral, Elsevier, Rio de Janeiro.

- SETOR SAÚDE (2015): Judicialização da saúde no Brasil: dados e experiência. Tomado de: https://setorsaude.com.br/estudo-revela-situacao-da-judicializacao-da-saude-nobrasil/, fecha de consulta: 19.08.19.

- MARTINI, Sandra Regina; STURZA, Janaína Machado (2019): Direitos Humanos: saúde e 
fraternidade, Evangraf, Porto Alegre.

- MASSA ARZABE, Patrícia Helena (2006): “Dimensão jurídica das políticas públicas” en BUCCl, Maria Paula Dallari (org.): Políticas públicas: reflexões sobre o conceito jurídico, Saraiva, São Paulo.

- MORAIS, José Luis Bolzan de; SPENGLER, Fabiana Marion (2019): Mediação e arbitragem: Alternativas à jurisdição, Livraria do Advogado, Porto Alegre.

- RIBEIRO, Wesllay Carlos (2018): "A mediação como meio de resolução de conflitos na área de saúde, en Revista De Direito Sanitário: Jornaul of Health Law, USP, São Paulo, v.18, n.3, nov.2017/feb.2018. Tomado de: https://www.revistas.usp.br/rdisan/article/ view/144648\&gt, fecha de consulta: 16.09.2019.

- FERNANDES, Antonio Joaquim Schellenberger (2018): “Mediação Sanitária: Os elementos materiais do direito coletivo à saúde", en FERNANDES, Antonio Joaquim Schellenberger: Direito à saúde: tutela coletiva e mediação sanitária, Editora D’Plácido, Belo Horizonte.

- SCHMIDT, João Pedro (2018): “Para estudar políticas públicas: aspectos conceituais, metodológicos e abordagens teóricas", en Revista do Direito, v. 3, n. 56, p. 119-149, sep/dic. 2018, Santa Cruz do Sul.

- SILVA, Pedro Luís Barros; MELO, Marcus André Barreto de (2000): O processo de implantação de políticas públicas no Brasil: características determinantes de avaliação de programas e projetos, en Cadernos de pesquisa, n. 48, Núcleos de Políticas Públicas, Unicamp.

- SPENGLER, Fabiana Marion (2017): Mediação de conflitos. Teoria e Prática, Livraria do Advogado, Porto Alegre.

- SPENGLER, Fabiana Marion (2019a): Dicionário de Mediação: Volume I, Essere nel Mondo, Santa Cruz do Sul.

- SPENGLER, Fabiana Marion (2019b): Dicionário de Mediação: Volume II, Essere nel Mondo, Santa Cruz do Sul.

- STEIN, Ernesto (2007): A política das políticas públicas: progresso econômicos e social na América Latina: relatório 2006, Banco Interamericano de Desenvolvimento e David Rockefeller Center for Latin America Studies, Havard University, Elsevier Washington, Rio de Janeiro. 